\title{
The Permanence and Extinction of the Single Species with Contraception Control and Feedback Controls
}

\author{
Qiuying Li, ${ }^{1}$ Fengqin Zhang, ${ }^{1}$ Xiaomei Feng, ${ }^{1}$ \\ Wenjuan Wang, ${ }^{1}$ and Kai Wang ${ }^{2}$ \\ ${ }^{1}$ Department of Applied Mathematics, Yuncheng University, Yuncheng 044000, China \\ ${ }^{2}$ Department of Medical Engineering and Technology, Xinjiang Medical University, Urumqi 830051, China
}

Correspondence should be addressed to Kai Wang, wangkaimath@sina.com

Received 8 February 2012; Accepted 3 April 2012

Academic Editor: Elena Braverman

Copyright (C) 2012 Qiuying Li et al. This is an open access article distributed under the Creative Commons Attribution License, which permits unrestricted use, distribution, and reproduction in any medium, provided the original work is properly cited.

Population control has become a major problem in many wildlife species. Sterility control through contraception has been proposed as a method for reducing population size. In this paper, the single species with sterility control and feedback controls is considered. Sufficient conditions are obtained for the permanence and extinction of the system. The results show that the feedback controls do not influence the permanence of the species.

\section{Introduction}

Control of wildlife pest populations has usually relied on methods like chemical pesticides, biological pesticides, remote sensing and measure, computers, atomic energy, and so forth. Some brilliant achievements have been obtained. However, the warfare will never be over. Although a large variety of pesticide were used to control wildlife pest populations, the wildlife pests impairing crops are increasing especially because of resistance to the pesticide. So the pesticides are invalid. Moreover, wildlife pests will continue. On the other hand, the chemical pesticide kills not only wildlife pests but also their natural enemies. Therefore, wildlife pests are rampant. Now, sterile control to suppress wildlife pests is one of the most important measures in wildlife pest control. Sterile control [1-5] is especially for the purpose of suppressing the abundance of the pest in a new target region to a level at which it no longer causes economic damage. This can be achieved by releasing sterile insects into the environment in very large numbers in order to mate with the native insects that are present in the environment. A native female that mates with a sterile male will produce eggs, but 
the eggs will not hatch (the same effect will occur for the reciprocal cross). If there is a sufficiently high number of sterile insects than most of the crosses are sterile, and as time goes on, the number of native insects decreases and the ratio of sterile to normal insects increases, thus driving the native population to extinction. Sterile male techniques were first used successfully in 1958 in Florida to control Screwworm fly (Cochliomya omnivorax). A number of mathematical models have been done to assist the effectiveness of the SIT (see, e.g., [2-4]). Recently, Liu and Li [6] considered the following contraception control model:

$$
\begin{aligned}
& x_{1}^{\prime}(t)=x_{1}(t)\left(b-k\left(x_{1}(t)+x_{2}(t)\right)-\mu\right) \\
& x_{2}^{\prime}(t)=\mu x_{1}(t)-\left[d+k\left(x_{1}(t)+x_{2}(t)\right)\right] x_{2}(t),
\end{aligned}
$$

where $x_{1}(t), x_{2}(t)$ represent, respectively, the density of the fertile species and the sterile species at time $t$. The authors proved the equilibrium point of system (1.1) is stable under appropriate conditions. In view of the effects of a periodically changing environment, we consider the following nonautonomous contraception model:

$$
\begin{aligned}
& x_{1}^{\prime}(t)=x_{1}(t)\left(b(t)-k(t)\left(x_{1}(t)+x_{2}(t)\right)-\mu(t)\right), \\
& x_{2}^{\prime}(t)=\mu(t) x_{1}(t)-\left[d(t)+k(t)\left(x_{1}(t)+x_{2}(t)\right)\right] x_{2}(t) .
\end{aligned}
$$

However, we note that ecosystem in the real world are continuously disturbed by unpredictable forces which can result in changes in the biological parameters such as survival rates. Of practical interest in ecosystem is the question of whether or not an ecosystem can withstand those unpredictable forces which persist for a finite period of time. In the language of control variables, we call the disturbance functions as control variables. So it is necessary to study models with control variables which are so-called disturbance functions, and to find some suitable conditions to prevent a particular species from dying out. In 1993, Gopalsamy and Weng [7] introduced a feedback control variable into the delay logistic model and discussed the asymptotic behavior of solution in logistic models with feedback controls, in which the control variables satisfy certain differential equation.

In recent years, the population dynamical systems with feedback controls have been studied in many articles, for example, see [7-12] and references cited therein. However, to the best of the authors knowledge, to this day, still less scholars consider the nonautonomous single species with contraception control and feedback controls.

Motivated by the above works, we focus our attention on the permanence of species for the following single species nonautonomous systems with delays and feedback control:

$$
\begin{aligned}
x_{1}^{\prime}(t) & =x_{1}(t)\left(b(t)-k(t)\left(x_{1}(t)+x_{2}(t)\right)-\mu(t)-c_{1}(t) u\left(t-\sigma_{1}(t)\right)\right), \\
x_{2}^{\prime}(t) & =\mu(t) x_{1}(t)-\left[d(t)+k(t)\left(x_{1}(t)+x_{2}(t)\right)+c_{2}(t) u\left(t-\sigma_{2}(t)\right)\right] x_{2}(t), \\
u^{\prime}(t) & =-e(t) u(t)+f_{1}(t) x_{1}\left(t-\tau_{1}(t)\right)+f_{2}(t) x_{2}\left(t-\tau_{2}(t)\right),
\end{aligned}
$$

where $x_{1}(t), x_{2}(t)$ represent, respectively, the density of the fertile population and the sterile population at time $t . u(t)$ is the control variable at time $t . b(t), k(t)$ represent, respectively, the intrinsic growth rate and density-dependent rate of the species at time $t$, respectively. $\mu(t)$ is the migration rates from the fertile population to the sterile population. The function $b(t)$ is bounded continuous defined on $R_{+}=[0, \infty)$; functions $\mu(t), k(t), d(t), e(t), c_{i}(t)$, 
$f_{i}(t), \sigma_{i}(t)$, and $\tau_{i}(t)(i=1,2)$ are continuous, bounded, and nonnegative defined on $R_{+}$. Let $\tau=\sup \left\{\tau_{1}(t), \tau_{2}(t), \sigma_{1}(t), \sigma_{2}(t): t \geq 0\right\}$.

We will consider (1.3) together with initial conditions:

$$
x_{i}(\theta)=\phi_{i}(\theta), \quad u(\theta)=\psi(\theta),
$$

where $\phi_{i}(\theta), \psi(\theta) \in B C^{+}[-\tau, 0]$ and

$$
B C^{+}[-\tau, 0]=\{\phi \in C([-\tau, 0],[0,+\infty]): \phi(0)>0, \phi(\theta) \text { is bounded }\} .
$$

By the fundamental theory of functional differential equations [12], it is not difficult to see that the solution $\left(x_{1}(t), x_{2}(t), u(t)\right)$ of $(1.3)$ is unique and positive if initial functions satisfy initial condition (1.4). So, in this paper, the solution of (1.3) satisfying initial conditions (1.4) is said to be positive.

The main purpose of this paper is to establish a new general criterion for the permanence and the extinction of (1.3), which is described by integral form and independent feedback controls. The paper is organized as follows. In the next section, we will give some assumptions and useful lemmas. In Section 3, some new sufficient conditions which guarantee the permanence of all positive solutions for (1.3) are obtained. In Section 4, we obtained some new sufficient conditions which guarantee the extinction of all positive solutions for (1.3).

\section{Preliminaries}

Throughout this paper, we will introduce the following assumptions:

$\left(\mathrm{H}_{1}\right)$ there exist constants $\omega_{i}>0(i=1,2)$ such that

$$
\liminf _{t \rightarrow \infty} \int_{t}^{t+\omega_{1}}[b(s)-\mu(s)] d s>0, \quad \quad \liminf _{t \rightarrow \infty} \int_{t}^{t+\omega_{2}} \mu(s) d s>0 ;
$$

$\left(\mathrm{H}_{2}\right)$ there exist constants $\lambda_{i}>0(i=1,2)$, such that

$$
\liminf _{t \rightarrow \infty} \int_{t}^{t+\lambda_{1}} k(s) d s>0, \quad \liminf _{t \rightarrow \infty} \int_{t}^{t+\lambda_{2}} d(s) d s>0 ;
$$

$\left(\mathrm{H}_{3}\right)$ there exists a constant $\gamma>0$ such that

$$
\liminf _{t \rightarrow \infty} \int_{t}^{t+r} e(s) d s>0
$$

In addition, for a function $g(t)$ defined on set $I \subset R$, we denote

$$
g^{L}=\inf _{t \in I} g(t), \quad g^{M}=\sup _{t \in I} g(t) .
$$


Now, we state several lemmas which will be useful in the proving of main results in this paper.

First, we consider the following nonautonomous logistic equation:

$$
x^{\prime}(t)=x(t)(r(t)-a(t) x(t))
$$

where functions $a(t), r(t)$ are bounded and continuous on $R_{+}$. Furthermore, $a(t) \geq 0$ for all $t \geq 0$. We have the following result which is given in [13] by Teng and Li.

Lemma 2.1. Suppose that there exist constants $\lambda, \sigma$ such that

$$
\liminf _{t \rightarrow \infty} \int_{t}^{t+\lambda} r(s) d s>0, \quad \quad \liminf _{t \rightarrow \infty} \int_{t}^{t+\sigma} a(s) d s>0
$$

Then,

(a) there exist positive constants $m$ and $M$ such that

$$
m \leq \liminf _{t \rightarrow \infty} x(t) \leq \limsup _{t \rightarrow \infty} x(t) \leq M
$$

for any positive solution $x(t)$ of (2.5);

(b) $\lim _{t \rightarrow \infty}\left(x^{(1)}(t)-x^{(2)}(t)\right)=0$ for any two positive solutions $x^{(1)}(t)$ and $x^{(2)}(t)$ of $(2.5)$.

Further, consider the following nonautonomous linear equation:

$$
u^{\prime}(t)=r(t)-e(t) u(t)
$$

where functions $r(t)$ and $e(t)$ are bounded continuous defined on $R_{+}$, and $r(t) \geq 0$ for all $t \geq 0$. One has the following result.

Lemma 2.2. Suppose that $\left(H_{3}\right)$ holds. Then,

(a) there exists a positive constant $U$ such that $\lim _{\sup } \operatorname{sum}_{t \rightarrow \infty} u(t) \leq U$ for any positive solution $u(t)$ of $(2.8)$;

(b) $\lim _{t \rightarrow \infty}\left(u^{(1)}(t)-u^{(2)}(t)\right)=0$ for any two positive solutions $u^{(1)}(t)$ and $u^{(2)}(t)$ of (2.8).

The proof of Lemma 2.2 is very simple by making a transformation with $u(t)=1 / x(t)$. This produces the calculations: $u^{\prime}(t)=-\left(1 / x^{2}(t)\right) x^{\prime}(t)$ and $x^{\prime}(t)=x(t)(e(t)-r(t) x(t))$. Then, according to the Lemma 2.1 one can obtain Lemma 2.2.

Lemma 2.3. Suppose that $\left(H_{3}\right)$ holds. Then, for any constants $\epsilon>0$ and $M>0$, there exist constants $\delta=\delta(\epsilon)>0$ and $T=T(\epsilon, M)>0$ such that for any $t_{0} \in R_{+}$and $u_{0} \in R$ with $\left|u_{0}\right| \leq M$, when $|r(t)|<\delta$ for all $t \geq t_{0}$, one has

$$
\left|u\left(t, t_{0}, u_{0}\right)\right|<\epsilon \quad \forall t \geq t_{0}+T
$$

where $u\left(t, t_{0}, u_{0}\right)$ is the solution of (2.8) with initial condition $u\left(t_{0}\right)=u_{0}$. 
The proof of Lemma 2.3 can be found as Lemma 2.4 in [11] by Wang et al.

\section{Main Results}

In this section, we study the permanence of species $x_{1}(t), x_{2}(t)$ of (1.3). First, we have the theorem on the ultimate boundedness of all positive solutions of (1.3).

Theorem 3.1. Suppose that assumptions $\left(H_{1}\right)-\left(H_{3}\right)$ hold. Then, any positive solution of (1.3) is ultimate bounded, in the sense that there exists a positive constant $M>0$ such that

$$
\limsup _{t \rightarrow \infty} x_{1}(t)<M, \quad \quad \limsup _{t \rightarrow \infty} x_{2}(t)<M, \quad \limsup _{t \rightarrow \infty} u(t)<M,
$$

for any positive solution $\left(x_{1}(t), x_{2}(t), u(t)\right)$ of $(1.3)$.

Proof. Let $\left(x_{1}(t), x_{2}(t), u(t)\right)$ be any positive solution of (1.3). We first prove that the component $x_{1}(t)$ of (1.3) is ultimately bounded. From the first equation of (1.3), we have

$$
\frac{d x_{1}(t)}{d t} \leq x_{1}(t)\left(b(t)-\mu(t)-k(t) x_{1}(t)\right)
$$

We consider the following auxiliary equation:

$$
\frac{d y(t)}{d t}=y(t)(b(t)-\mu(t)-k(t) y(t))
$$

then by $\left(\mathrm{H}_{1}\right)$ and applying Lemma 2.1 , there exists a constant $M_{1}$ such that

$$
\limsup _{t \rightarrow \infty} y(t)<M_{1}
$$

for any positive solution $y(t)$ of (3.3). Let $y^{*}(t)$ be the solution of (3.3) satisfying initial condition $y^{*}\left(t_{0}\right)=x_{1}\left(t_{0}\right)$. Further, from comparison theorem, it follows that

$$
x_{1}(t)<y^{*}(t) \quad \forall t>t_{0}
$$

Thus, we finally obtain that

$$
\limsup _{t \rightarrow \infty} x_{1}(t)<M_{1}
$$

From inequality (3.6), we obtain that there exists a positive constant $T_{1}$ such that

$$
x_{1}(t)<M_{1} \quad \forall t \geq T_{1} .
$$


Hence, from the second equation of (1.3), one has

$$
\frac{d x_{2}(t)}{d t} \leq \mu(t) M_{1}-d(t) x_{2}(t)
$$

for all $t \geq T_{1}$. Further, consider the following auxiliary equation:

$$
\frac{d v(t)}{d t}=\mu(t) M_{1}-d(t) v(t)
$$

from assumptions $\left(\mathrm{H}_{1}\right)$ and $\left(\mathrm{H}_{2}\right)$ and according to Lemma 2.2, there exists constant $M_{2}>0$ such that

$$
\limsup _{t \rightarrow \infty} v(t)<M_{2}
$$

for the solution $v(t)$ of (3.9) with initial condition $v\left(T_{1}\right)=x_{2}\left(T_{1}\right)$. By the comparison theorem, we have

$$
x_{2}(t) \leq v(t) \quad \forall t \geq T_{1} .
$$

From this, we further obtain

$$
\limsup _{t \rightarrow \infty} x_{2}(t)<M_{2}
$$

Then, we obtain that there exists constant $T_{2}>T_{1}$ such that

$$
x_{2}(t)<M_{2} \quad \forall t \geq T_{2} .
$$

From the third equation of (1.3), we have

$$
\frac{d u(t)}{d t} \leq-e(t) u(t)+M_{1} f_{1}(t)+M_{2} f_{2}(t)
$$

for all $t \geq T_{2}+\tau$. Consider the following auxiliary equation:

$$
\frac{d v(t)}{d t}=-e(t) v(t)+M_{1} f_{1}(t)+M_{2} f_{2}(t)
$$

By assumption $\left(\mathrm{H}_{3}\right)$ and conclusions of Lemma 2.2, we can get that there exists a constant $M_{3}>0$ such that

$$
\limsup _{t \rightarrow \infty} v(t)<M_{3}
$$


for the solution $v(t)$ of (3.15) with initial condition $v\left(T_{2}+\tau\right)=u\left(T_{2}+\tau\right)$. By the comparison theorem, we have

$$
u(t) \leq v(t) \quad \forall t \geq T_{2}+\tau
$$

Hence, we further obtain

$$
\limsup _{t \rightarrow \infty} u(t)<M_{3}
$$

Choose the constant $M=\max \left\{M_{1}, M_{2}, M_{3}\right\}$, then we finally obtain

$$
\limsup _{t \rightarrow \infty} x_{1}(t)<M, \quad \quad \limsup _{t \rightarrow \infty} x_{2}(t)<M, \quad \limsup _{t \rightarrow \infty} u(t)<M .
$$

This completes the proof.

Theorem 3.2. Suppose that assumptions $\left(H_{1}\right)-\left(H_{3}\right)$ hold. Then, there exists a constant $\eta>0$, which is independent of any solution of (1.3), such that

$$
\liminf _{t \rightarrow \infty} x_{1}(t)>\eta, \quad \liminf _{t \rightarrow \infty} x_{2}(t)>\eta,
$$

for any positive solution $\left(x_{1}(t), x_{2}(t), u(t)\right)$ of (1.3).

Proof. Let $\left(x_{1}(t), x_{2}(t), u(t)\right)$ be a solution of (1.3) satisfying initial condition (1.4). In view of Theorem 3.1, there exists a $T_{0}$ such that for all $t>T_{0}$ we have $x_{i}(t)<M, u(t)<M(i=1,2)$. According to $\left(\mathrm{H}_{1}\right)$, we can choose constants $\varepsilon_{0}>0$ and $T_{1}>0$ such that, for all $t \geq T_{1}$, we have

$$
\int_{t}^{t+\omega_{1}}\left(b(s)-\mu(s)-2 k(s) \varepsilon_{0}-c_{1}(s) \varepsilon_{0}\right) d s>\varepsilon_{0}
$$

Next, we consider the following equation:

$$
\frac{d u(t)}{d t}=-e(t) u(t)+f_{1}(t) \alpha_{0}+f_{2}(t) \alpha_{0}
$$

where $\alpha_{0}$ was given in the later. By $\left(\mathrm{H}_{3}\right)$, we have (3.22) satisfying all the conditions of Lemma 2.3. So, we can obtain that, for given constants $\varepsilon_{0}>0$ and $M>0$ ( $M$ was given in Theorem 3.1), there exist constants $\delta_{0}=\delta_{0}\left(\varepsilon_{0}\right)>0$ and $T^{*}=T^{*}\left(\varepsilon_{0}, M\right)>0$ such that for any $t_{0} \in R_{+}$and $0 \leq u_{0} \leq M$, when $\alpha_{0} f_{1}(t)+\alpha_{0} f_{2}(t)<\delta_{0}$ for all $t \geq t_{0}$, we have

$$
u\left(t, t_{0}, u_{0}\right)<\varepsilon_{0} \quad \forall t \geq t_{0}+T^{*},
$$

where $u\left(t, t_{0}, u_{0}\right)$ is the solution of (3.22) with initial condition $u\left(t_{0}\right)=u_{0}$. 
Further, consider the following equation:

$$
\frac{d z(t)}{d t}=\mu(t) \alpha_{0}-d(t) z(t)
$$

where $\alpha_{0}$ was given in (3.22). By $\left(\mathrm{H}_{2}\right)$, we have (3.24) satisfying all the conditions of Lemma 2.3, so by Lemma 2.3, for given constants $\alpha_{0}>0$ and $M>0$, there exist constants $\delta_{1}=\delta_{1}\left(\alpha_{0}\right)>0$ and $T^{* *}=T^{* *}\left(\alpha_{0}, M\right)>0$ such that for any $t_{0} \in R_{+}$and $0 \leq z_{0} \leq M$, when $\mu(t) \alpha_{0}<\delta_{1}$ for all $t \geq t_{0}$, we have

$$
z\left(t, t_{0}, z_{0}\right)<\alpha_{0} \quad \forall t \geq t_{0}+T^{* *},
$$

where $z\left(t, t_{0}, z_{0}\right)$ is the solution of (3.24) with initial condition $z\left(t_{0}\right)=z_{0}$.

Let $\alpha_{0} \leq \min \left\{\varepsilon_{0},\left(\delta_{1} /\left(\mu^{M}+1\right)\right),\left(\delta_{0} /\left(f_{1}^{M}+f_{2}^{M}+1\right)\right)\right\}$ such that for all $t \geq T_{1}$, we have

$$
\int_{t}^{t+\omega_{1}}\left(b(s)-\mu(s)-2 k(s) \alpha_{0}-f_{1}(s) \varepsilon_{0}\right) d s>\varepsilon_{0}
$$

We first prove that

$$
\limsup _{t \rightarrow \infty} x_{1}(t) \geq \alpha_{0}
$$

In fact, if (3.27) is not true, then there exist a positive solution $\left(x_{1}(t), x_{2}(t), u(t)\right)$ of $(1.3)$ and a constant $T_{2} \geq T_{1}$ such that

$$
x_{1}(t)<\alpha_{0}
$$

for all $t \geq T_{2}$.

From the second equation of (1.3), we have

$$
\frac{d x_{2}(t)}{d t} \leq \mu(t) \alpha_{0}-d(t) x_{2}(t)
$$

for all $t \geq T_{2}$. Let $\widehat{z}(t)$ be the solution of (3.24) with initial condition $\widehat{z}\left(T_{2}\right)=x_{2}\left(T_{2}\right)$, by the comparison theorem, we have $x_{2}(t) \leq \hat{z}(t)$ for all $t \geq T_{2}$. In (3.25), we choose $t_{0}=T_{2}$ and $\widehat{z}_{0}=x_{2}\left(T_{2}\right)$, since $\mu(t) \alpha_{0}<\delta_{1}$, we obtain $\widehat{z}\left(t, t_{0}, \widehat{z}_{0}\right)<\alpha_{0}$ for all $t \geq T_{2}+T^{* *}$. Hence, we further obtain

$$
x_{2}\left(t, T_{2}, x_{2}\left(T_{2}\right)\right)<\alpha_{0} \quad \forall t \geq T_{2}+T^{* *} .
$$

By applying (3.28) and (3.30) to the third equation of (1.3), it follows that

$$
\frac{d u(t)}{d t} \leq-e(t) u(t)+f_{1}(t) \alpha_{0}+f_{2}(t) \alpha_{0}
$$


for all $t \geq T_{2}+T^{* *}+\tau$. Let $\widehat{u}(t)$ be the solution of (3.22) with initial condition $\widehat{u}\left(T_{2}+T^{* *}+\tau\right)=$ $u\left(T_{2}+T^{* *}+\tau\right)$, by the comparison theorem, we have

$$
u(t) \leq \widehat{u}(t) \quad \forall t \geq T_{2}+T^{* *}+\tau .
$$

In (3.23), we choose $t_{0}=T_{2}+T^{* *}+\tau$ and $\widehat{u}_{0}=u\left(T_{2}+T^{* *}+\tau\right)$, since $\alpha_{0} f_{1}(t)+\alpha_{0} f_{2}(t)<\delta_{0}$ for all $t \geq T_{2}+T^{* *}+\tau$, we obtain

$$
\widehat{u}\left(t, T_{2}+T^{* *}+\tau, u\left(T_{2}+T^{* *}+\tau\right)\right)<\varepsilon_{0} \quad \forall t \geq T_{2}+T^{* *}+\tau+T^{*} .
$$

Hence, from (3.32), we further obtain

$$
u\left(t, T_{2}+T^{* *}+\tau, u\left(T_{2}+T^{* *}+\tau\right)\right)<\varepsilon_{0} \quad \forall t \geq T_{2}+T^{* *}+\tau+T^{*} .
$$

Hence, by (3.30) and (3.34) it follows that

$$
\frac{d x_{1}(t)}{d t} \geq x_{1}(t)\left(b(t)-\mu(t)-2 k(t) \alpha_{0}-c_{1}(t) \varepsilon_{0}\right)
$$

for any $t>T_{2}+T^{* *}+T^{*}+2 \tau$. Integrating (3.35) from $T_{3}=T_{2}+T^{* *}+T^{*}+2 \tau$ to $t$ we have

$$
x_{1}(t) \geq x_{1}\left(T_{3}\right) \exp \int_{T_{3}}^{t}\left(b(s)-\mu(s)-2 k(s) \alpha_{0}-c_{1}(s) \varepsilon_{0}\right) d s .
$$

Thus, from (3.26), we have $x_{1}(t) \rightarrow \infty$ as $t \rightarrow \infty$, which leads to a contradiction. So, (3.27) holds.

Now, we prove the conclusion of Theorem 3.2. In fact, if it is not true, then there exists an initial functions sequence $\left\{Z^{(m)}\right\}=\left\{\left(\phi_{1}^{(m)}(\theta), \phi_{2}^{(m)}(\theta), \psi^{(m)}(\theta)\right)\right\}, \theta \in[-\tau, 0]$ such that

$$
\liminf _{t \rightarrow \infty} x_{1}\left(t, Z^{(m)}\right)<\frac{\alpha_{0}}{(m+1)^{2}} \quad \forall m=1,2, \ldots,
$$

for the solution $\left(x_{1}\left(t, Z^{(m)}\right), x_{2}\left(t, Z^{(m)}\right), u\left(t, Z^{(m)}\right)\right)$ of (1.3). From (3.27) and (3.37), for every $m$, there are two time sequences $\left\{s_{q}^{(m)}\right\}$ and $\left\{t_{q}^{(m)}\right\}$, satisfying $0<s_{1}^{(m)}<t_{1}^{(m)}<s_{2}^{(m)}<t_{2}^{(m)}<$ $\cdots<s_{q}^{(m)}<t_{q}^{(m)}<\cdots$ and $\lim _{q \rightarrow \infty} s_{q}^{(m)}=\infty$, such that

$$
\begin{gathered}
x_{1}\left(s_{q}^{(m)}, Z^{(m)}\right)=\frac{\alpha_{0}}{m+1}, \quad x_{1}\left(t_{q}^{(m)}, Z^{(m)}\right)=\frac{\alpha_{0}}{(m+1)^{2}}, \\
\frac{\alpha_{0}}{(m+1)^{2}} \leq x_{1}\left(t, Z^{(m)}\right) \leq \frac{\alpha_{0}}{m+1} \quad \forall t \in\left(s_{q}^{(m)}, t_{q}^{(m)}\right) .
\end{gathered}
$$


From Theorem 3.1, we can choose a positive constant $T^{(m)}$ such that $x_{1}\left(t, Z^{(m)}\right)<M$ and $x_{2}\left(t, Z^{(m)}\right)<M$ and $u\left(t, Z^{(m)}\right)<M$, for all $t>T^{(m)}$. Further, there is an integer $K_{1}^{(m)}>0$ such that $s_{q}^{(m)}>T^{(m)}+\tau$ for all $q \geq K_{1}^{(m)}$. Let $q \geq K_{1}^{(m)}$, for any $t \in\left(s_{q}^{(m)}, t_{q}^{(m)}\right)$, we have

$$
\begin{aligned}
\frac{d x_{1}\left(t, Z^{(m)}\right)}{d t} & \geq x_{1}\left(t, Z^{(m)}\right)\left[b(t)-\mu(t)-k(t) M-k(t) M-c_{1}(t) M\right] \\
& \geq-\gamma_{1} x_{1}\left(t, Z^{(m)}\right)
\end{aligned}
$$

where $\gamma_{1}=\sup _{t \in R_{+}}\left\{\left|b(t)-\mu(t)-2 k(t) M-c_{1}(t) M\right|\right\}$. Integrating the above inequality from $s_{q}^{(m)}$ to $t_{q}^{(m)}$, we further have

$$
\begin{aligned}
\frac{\alpha_{0}}{(m+1)^{2}} & =x_{1}\left(t_{q}^{(m)}, Z^{(m)}\right) \geq x_{1}\left(s_{q}^{(m)}, Z^{(m)}\right) \exp \left[-\gamma_{1}\left(t_{q}^{(m)}-s_{q}^{(m)}\right)\right] \\
& =\frac{\alpha_{0}}{m+1} \exp \left[-\gamma_{1}\left(t_{q}^{(m)}-s_{q}^{(m)}\right)\right] .
\end{aligned}
$$

Consequently,

$$
t_{q}^{(m)}-s_{q}^{(m)} \geq \frac{\ln (m+1)}{\gamma_{1}} \quad \forall q \geq K_{1}^{(m)}, m=1,2, \ldots
$$

we can choose a large enough $N_{0}$ such that

$$
t_{q}^{(m)}-s_{q}^{(m)} \geq T^{* *}+T^{*}+2 \tau+\omega_{1} \quad \forall m \geq N_{0}, q \geq K_{1}^{(m)}
$$

For any $m \geq N_{0}, q \geq K_{1}^{(m)}$, and $t \in\left[s_{q}^{(m)}, t_{q}^{(m)}\right]$, from (3.39), we can obtain

$$
\begin{aligned}
\frac{d x_{2}\left(t, Z^{(m)}\right)}{d t} & \leq \mu(t) \frac{\alpha_{0}}{m+1}-d(t) x_{2}\left(t, Z^{(m)}\right) \\
& \leq \mu^{M} \alpha_{0}-d(t) x_{2}\left(t, Z^{(m)}\right) .
\end{aligned}
$$

Let $\widehat{z}(t)$ be solution of (3.24) with initial condition $\widehat{z}\left(s_{q}^{(m)}\right)=x_{2}\left(s_{q}^{(m)}\right)$, by the comparison theorem, we have $x_{2}(t) \leq \widehat{z}(t)$ for all $t \geq s_{q}^{(m)}$. In (3.25), we choose $t_{0}=s_{q}^{(m)}$ and $\widehat{z}_{0}=z\left(s_{q}^{(m)}\right)$, since $\mu^{M} \alpha_{0}<\delta_{1}$ and $x_{2}\left(s_{q}^{(m)}<M\right.$, we obtain $\widehat{z}\left(t, s_{q}^{(m)}, z\left(s_{q}^{(m)}\right)\right)<\alpha_{0}$ for all $t \geq s_{q}^{(m)}+T^{* *}$. By Lemma 2.3, we can obtain that $T^{* *}=T^{* *}\left(\alpha_{0}, M\right)$ is independent of $m$. Hence, we further obtain

$$
x_{2}\left(t, s_{q}^{(m)}, x_{2}\left(s_{q}^{(m)}\right)\right)<\alpha_{0}
$$


for all $t>s_{q}^{(m)}+T^{* *}$. From the third equation of (1.3), we obtain

$$
\begin{aligned}
\frac{d u\left(t, Z^{(m)}\right)}{d t} & =-e(t) u\left(t, Z^{(m)}\right)+f_{1}(t) x_{1}\left(t-\tau_{1}(t), Z^{(m)}\right)+f_{2}(t) x_{2}\left(t-\tau_{2}(t), Z^{(m)}\right) \\
& \leq-e(t) u\left(t, Z^{(m)}\right)+f_{1}(t) \alpha_{0}+f_{2}(t) \alpha_{0}
\end{aligned}
$$

for all $t \geq s_{q}^{(m)}+T^{* *}+\tau$. Assume that $\tilde{u}(t)$ is the solution of (3.22) with initial condition $\tilde{u}\left(s_{q}^{(m)}+T^{* *}+\tau\right)=u\left(s_{q}^{(m)}+T^{* *}+\tau\right)$, then we have

$$
u\left(t, Z^{(m)}\right) \leq \tilde{u}(t) \quad \forall t \in\left[s_{q}^{(m)}+T^{* *}+\tau, t_{q}^{(m)}\right], m \geq N_{0}, q \geq K_{1}^{(m)} .
$$

In (3.23), we choose $t_{0}=s_{q}^{(m)}+T^{* *}+\tau$ and $u_{0}=u\left(s_{q}^{(m)}+T^{* *}+\tau\right)$. Obviously, $\alpha_{0}\left(f_{1}(t)+f_{2}(t)\right)<\delta_{0}$ for all $t \geq s_{q}^{(m)}+T^{* *}+\tau$. So, we have

$$
\widetilde{u}(t)=\tilde{u}\left(t, s_{q}^{(m)}+T^{* *}+\tau, u\left(s_{q}^{(m)}+T^{* *}+\tau\right)\right)<\varepsilon_{0}
$$

for all $t \in\left[s_{q}^{(m)}+T^{* *}+\tau+T^{*}, t_{q}^{(m)}\right]$. Using the comparison theorem, it follows that

$$
u\left(t, Z^{(m)}\right)<\varepsilon_{0}
$$

for all $t \in\left[s_{q}^{(m)}+T^{* *}+\tau+T^{*}, t_{q}^{(m)}\right], q \geq K_{1}^{(m)}$, and $m \geq N_{0}$.

So, for any $m \geq N_{0}, q \geq K_{1}^{(m)}$, and $t \in\left[s_{q}^{(m)}+T^{* *}+T^{*}+2 \tau, t_{q}^{(m)}\right]$, from (3.26), (3.45), and (3.49), it follows

$$
\begin{aligned}
\frac{d x_{1}\left(t, Z^{(m)}\right)}{d t} & =x_{1}\left(t, Z^{(m)}\right)\left(b(t)-\mu(t)-k(t)\left(x_{1}\left(t, Z^{(m)}\right)+x_{2}\left(t, Z^{(m)}\right)\right)-c_{1}(t) u\left(t-\sigma_{1}(t), Z^{(m)}\right)\right) \\
& \geq x_{1}\left(t, Z^{(m)}\right)\left(b(t)-\mu(t)-2 k(t) \alpha_{0}-c_{1}(t) \varepsilon_{0}\right) .
\end{aligned}
$$

Integrating the above inequality from $t_{q}^{(m)}-\omega_{1}$ to $t_{q}^{(m)}$, then from (3.26), we obtain

$$
\begin{aligned}
\frac{\alpha_{0}}{(m+1)^{2}} & =x_{1}\left(t_{q}^{(m)}, Z^{(m)}\right) \\
& \geq x_{1}\left(t_{q}^{(m)}-\omega_{1}, Z^{(m)}\right) \exp \int_{t_{q}^{(m)}-\omega_{1}}^{t_{q}^{(m)}}\left(b(t)-\mu(t)-2 k(t) \alpha_{0}-c_{1}(t) \varepsilon_{0}\right) d t \\
& \geq \frac{\alpha_{0}}{(m+1)^{2}} \exp \left(\alpha_{0}\right)>\frac{\alpha_{0}}{(m+1)^{2}},
\end{aligned}
$$


which leads to a contradiction. Therefore, this contradiction shows that there exists constant $\eta_{1}>0$ such that

$$
\liminf _{t \rightarrow \infty} x_{1}(t)>\eta_{1}
$$

for any positive solution $\left(x_{1}(t), x_{2}(t), u(t)\right)$ of (1.3). Therefore, there exists constant $\eta_{2}>0$ such that

$$
\liminf _{t \rightarrow \infty} x_{2}(t)>\eta_{2}
$$

for any positive solution $\left(x_{1}(t), x_{2}(t), u(t)\right)$ of (1.3). This completes the proof.

Remark 3.3. In Theorem 3.2, we note that $\left(\mathrm{H}_{1}\right)-\left(\mathrm{H}_{3}\right)$ are decided by (1.3), which is independent of the feedback controls. So, the feedback controls have no influence on the permanence of (1.3).

\section{Extinction}

In this section, we discuss the extinction of the component $x_{1}(t), x_{2}(t)$ of (1.3).

Theorem 4.1. Suppose that there exist constants $\sigma_{1}, \sigma_{2}$, such that

$$
\limsup _{n \rightarrow \infty} \int_{t}^{t+\sigma_{1}}(b(s)-\mu(s)) d s<0 ; \quad \liminf _{t \rightarrow \infty} \int_{t}^{t+\sigma_{2}} k(s) d s>0
$$

hold. Then,

$$
\lim _{t \rightarrow \infty} x_{1}(t)=0, \quad \lim _{t \rightarrow \infty} x_{2}(t)=0,
$$

for any positive solution $\left(x_{1}(t), x_{2}(t), u(t)\right)$ of $(1.3)$.

Proof. By the condition, for every given positive constant $\varepsilon$, there exist constants $\varepsilon_{1}$ and $T_{1}$ such that

$$
\int_{t}^{t+\sigma_{1}}(b(s)-\mu(s)-k(s) \varepsilon) d s<-\varepsilon_{1}
$$

for all $t>T_{1}$. First, we show that there exists a $T_{2}>T_{1}$, such that $x_{1}\left(T_{2}\right)<\varepsilon$. Otherwise, we have

$$
x_{1}(t) \geq \varepsilon, \quad \forall t>T_{1} .
$$

Hence, for all $t \geq T_{1}$, one has

$$
x_{1}^{\prime}(t)<x_{1}(t)(b(t)-k(t) \varepsilon-\mu(t)) .
$$


Thus, as $t \rightarrow+\infty$, we have

$$
\begin{aligned}
\varepsilon & \leq x_{1}(t)<x_{1}\left(T_{1}\right) \exp \left\{\int_{T_{1}}^{t}(b(s)-\mu(s)-k(s) \varepsilon) d s\right\} \\
& <x_{1}\left(T_{1}\right) \exp \left\{-\varepsilon_{1}\left(t-T_{1}\right)\right\} \longrightarrow 0 .
\end{aligned}
$$

So, $\varepsilon<0$, which leads to a contradiction. Therefore, there exists a $T_{2}>T_{1}$, such that $x_{1}\left(T_{2}\right)<\varepsilon$. Second, we show that

$$
x_{1}(t)<\varepsilon \exp \left\{\mu_{1} \sigma_{1}\right\} \quad \forall t>T_{2}
$$

where $\mu_{1}=\max _{t \in[0,+\infty]}\{b(t)+\mu(t)+k(t) \varepsilon\}$. Otherwise, there exists a $T_{3}>T_{2}$, such that $x_{1}\left(T_{3}\right)>$ $\varepsilon \exp \left\{\mu_{1} \sigma_{1}\right\}$. By the continuity of $x_{1}(t)$, there must exist $T_{4} \in\left(T_{2}, T_{3}\right)$ such that $x_{1}\left(T_{4}\right)=\varepsilon$ and $x_{1}(t)>\varepsilon$ for $t \in\left(T_{4}, T_{3}\right)$. Let $P_{1}$ be the nonnegative integer such that $T_{3} \in\left(T_{4}+P_{1} \sigma_{1}, T_{4}+\left(P_{1}+\right.\right.$ 1) $\left.\sigma_{1}\right)$. Further, we obtain that

$$
\begin{aligned}
\varepsilon \exp \left\{\mu_{1} \sigma_{1}\right\} & <x_{1}\left(T_{3}\right)<x_{1}\left(T_{4}\right) \exp \left\{\int_{T_{4}}^{T_{3}}(b(s)-\mu(s)-k(s) \varepsilon) d s\right\} \\
= & x_{1}\left(T_{4}\right) \exp \left\{\int_{T_{4}}^{T_{4}+P_{1} \sigma_{1}}(b(s)-\mu(s)-k(s) \varepsilon) d s\right. \\
& \left.\quad+\int_{T_{4}+P_{1} \sigma_{1}}^{T_{3}}(b(s)-\mu(s)-k(s) \varepsilon) d s\right\} \\
& <\varepsilon \exp \left\{\mu_{1} \sigma_{1}\right\},
\end{aligned}
$$

which leads to contradiction. This shows that (4.7) holds. By the arbitrariness of $\varepsilon$, it immediately follows that $x_{1}(t) \rightarrow 0$ as $t \rightarrow+\infty$. Further, we can obtain that $x_{2}(t) \rightarrow 0$ as $t \rightarrow+\infty$. This completes the proof of Theorem 4.1.

\section{Acknowledgment}

This work is supported by the National Natural Science Foundation of China (11071283), Research Project supported by Shanxi Scholarship Council of China (2011-093) and the Sciences Foundation of Shanxi (2009011005-3).

\section{References}

[1] R. J. Jackson, D. J. Maguire, and L. A. Hinds, “Infertility in mice induced by a recombinant ectromelia virus expressing mouse zona pellucida glycoprotein," Biology of Reproduction, vol. 58, pp. 152-159, 1998.

[2] A. T. Rutberga, R. E. Nauglea et al., "Effects of immunocontraception on a suburban popu- lation of white-tailed deer Odocoileus virginianus," Biological Conservation, vol. 116, pp. 243-250, 2004.

[3] F. Courchamp and S. J. Cornell, "Virus-vectored immunocontraception to control feral cats on islands: a mathematical model," Journal of Applied Ecology, vol. 37, pp. 903-913, 2000. 
[4] G. M. Hood, P. Chesson, and R. P. Pech, "Biological control using sterilizing viruses: host suppression and competition between viruses in non-spatial models," Journal of Applied Ecology, vol. 37, pp. 914925, 2000.

[5] Z. Zhang, "Mathematical models of wildlife management by contraception," Ecological Modelling, vol. 132, pp. 105-113, 2000.

[6] H. Liu and Q. Li, "Model of single-species population under contraceptive control and lethal control," Mathematics in Practice and Theory, vol. 39, no. 15, pp. 106-109, 2009.

[7] K. Gopalsamy and P. X. Weng, "Feedback regulation of logistic growth," International Journal of Mathematics and Mathematical Sciences, vol. 16, no. 1, pp. 177-192, 1993.

[8] Z. Teng, "Persistence and stability in general nonautonomous single-species Kolmogorov systems with delays," Nonlinear Analysis. Real World Applications, vol. 8, no. 1, pp. 230-248, 2007.

[9] H.-F. Huo and W.-T. Li, "Positive periodic solutions of a class of delay differential system with feedback control," Applied Mathematics and Computation, vol. 148, no. 1, pp. 35-46, 2004.

[10] F. Yin and Y. Li, "Positive periodic solutions of a single species model with feedback regulation and distributed time delay," Applied Mathematics and Computation, vol. 153, no. 2, pp. 475-484, 2004.

[11] K. Wang, Z. Teng, and H. Jiang, "On the permanence for $n$-species non-autonomous Lotka-Volterra competitive system with infinite delays and feedback controls," International Journal of Biomathematics, vol. 1, no. 1, pp. 29-43, 2008.

[12] F. Chen, J. Yang, L. Chen, and X. Xie, "On a mutualism model with feedback controls," Applied Mathematics and Computation, vol. 214, no. 2, pp. 581-587, 2009.

[13] Z. Teng and Z. Li, "Permanence and asymptotic behavior of the $N$-species nonautonomous LotkaVolterra competitive systems," Computers and Mathematics with Applications, vol. 39, no. 7-8, pp. 107116, 2000. 


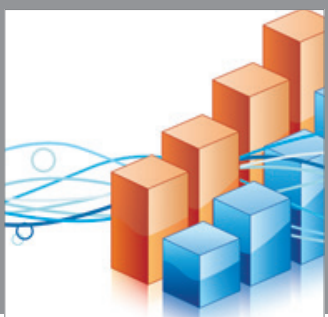

Advances in

Operations Research

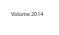

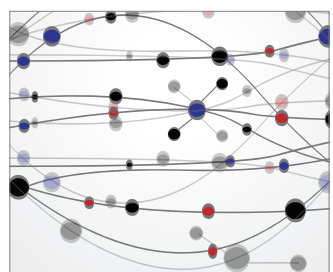

\section{The Scientific} World Journal
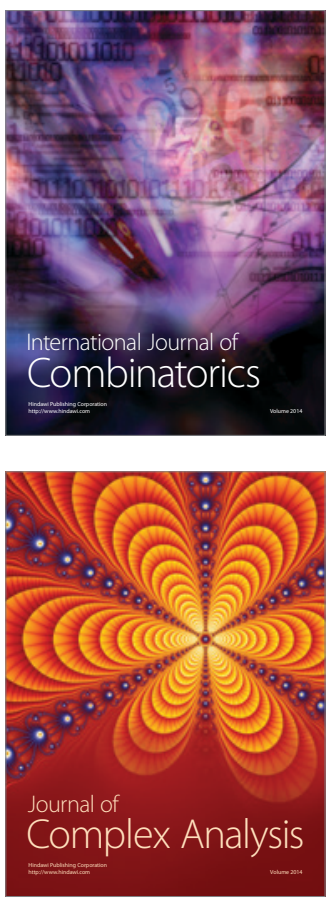

International Journal of

Mathematics and

Mathematical

Sciences
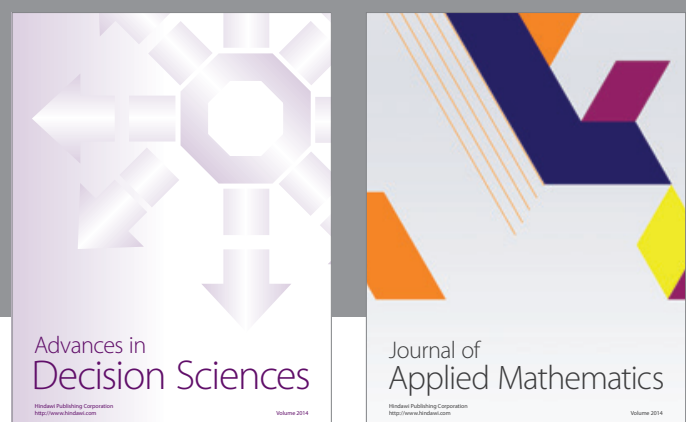

Journal of

Applied Mathematics
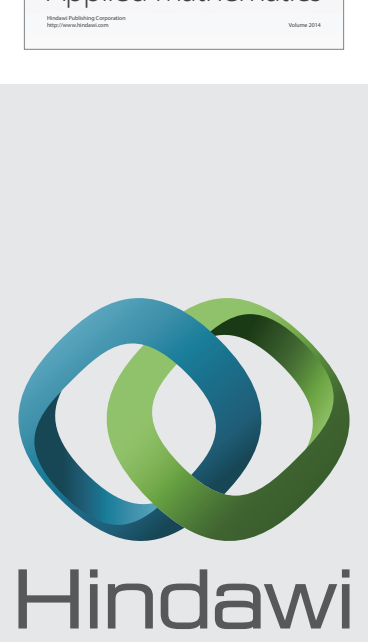

Submit your manuscripts at http://www.hindawi.com
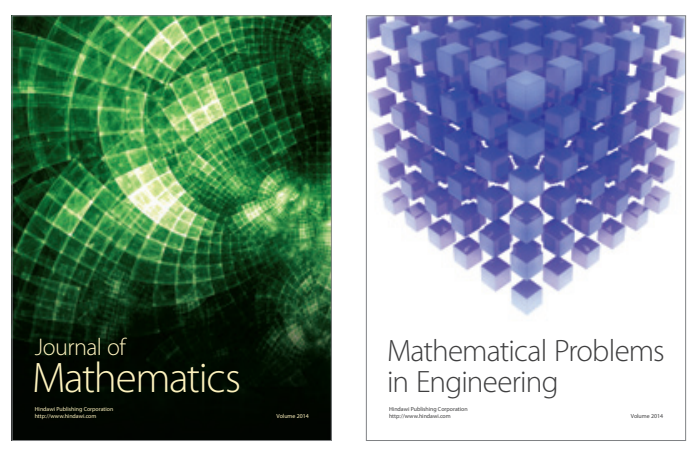

Mathematical Problems in Engineering
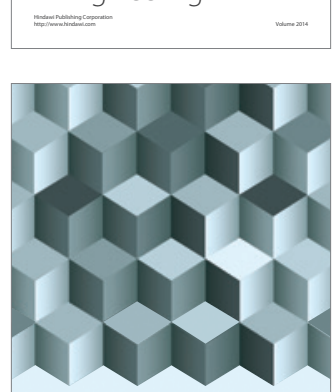

Journal of

Function Spaces
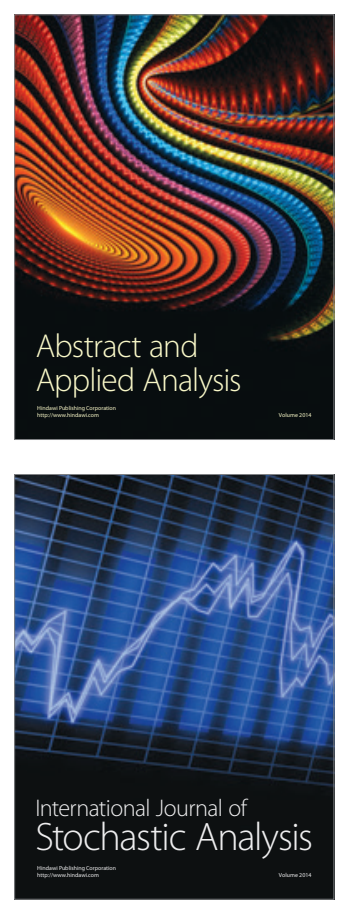

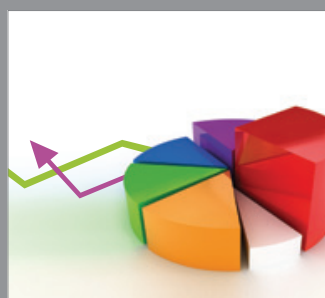

ournal of

Probability and Statistics

Promensencen
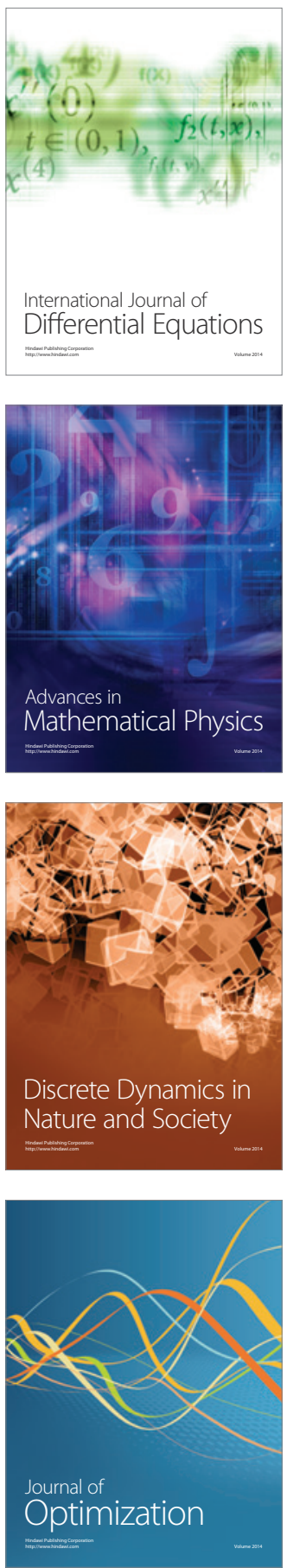